\title{
Suppression and recovery of the ferroelectric phase in multiferroic $\mathrm{MnWO}_{4}$
}

\author{
R. P. Chaudhury, ${ }^{1}$ B. Lorenz, ${ }^{1}$ Y. Q. Wang, ${ }^{1}$ Y. Y. Sun, ${ }^{1}$ and C. W. Chu $u^{1,2,3}$ \\ ${ }^{1}$ TCSUH and Department of Physics, University of Houston, Houston, Texas 77204-5002, USA \\ ${ }^{2}$ Lawrence Berkeley National Laboratory, 1 Cyclotron Road, Berkeley, California 94720, USA \\ ${ }^{3}$ Hong Kong University of Science and Technology, Hong Kong, China
}

(Received 15 January 2008; published 7 March 2008)

\begin{abstract}
We report the discovery of a complete suppression of ferroelectricity in $\mathrm{MnWO}_{4}$ by $10 \%$ iron substitution and its restoration in external magnetic fields. The spontaneous polarization in $\mathrm{Mn}_{0.9} \mathrm{Fe}_{0.1} \mathrm{WO}_{4}$ arises below $12 \mathrm{~K}$ in external fields above $4 \mathrm{~T}$. The magnetic and/or ferroelectric phase diagram is constructed from the anomalies of the dielectric constant, polarization, magnetization, and heat capacity. The observations are qualitatively described by a mean-field model with competing interactions and strong anisotropy. We propose that the magnetic field induces a noncollinear inversion symmetry breaking magnetic structure in $\mathrm{Mn}_{0.9} \mathrm{Fe}_{0.1} \mathrm{WO}_{4}$.
\end{abstract}

DOI: 10.1103/PhysRevB.77.104406

PACS number(s): 77.80.-e, 75.30.-m, 75.30.Kz, 75.50.Ee

\section{INTRODUCTION}

Multiferroic materials in which ferroelectric (FE) and magnetic orders coexist and mutually interact have recently attracted attention because of many novel physical phenomena observed in these compounds as well as their potential for applications as magnetoelectric sensors or memory chips. ${ }^{1,2}$ Common features and magnetoelectric properties of compounds with quite different chemical compositions and structures indicate a universal physical mechanism behind the multiferroic phenomena of a large materials class. It was shown that a noncollinear (or helical) spin density wave can break the spatial inversion symmetry and, with sufficiently strong spin-lattice coupling, can result in a lattice distortion with a FE polarization. ${ }^{3}$ The helical magnetic order was in fact observed in the FE phases of $\mathrm{TbMnO}_{3},{ }^{4} \mathrm{Ni}_{3} \mathrm{~V}_{2} \mathrm{O}_{8},{ }^{5}$ $\mathrm{MnWO}_{4}{ }^{6}$ etc. Most multiferroics exhibit highly frustrated magnetic orders due to geometric constraints or competing interactions. Local lattice distortions can therefore release the magnetic frustration, lower the magnetic energy, and induce local electrical dipoles that may add up to a macroscopic polarization if permitted by symmetry. ${ }^{7}$ In frustrated magnetic systems, several magnetic states are close in energy and compete for the ground state. As a result, in multiferroic systems, the FE polarization, which is induced by magnetic order, is easily controlled by small perturbations affecting the microscopic exchange constants or the magnetic order. For example, is was shown that magnetic fields ${ }^{6,8-10}$ and external pressure $^{11-13}$ have a significant effect on the ferroelectric polarization and the multiferroic properties.

The multiferroic $\mathrm{MnWO}_{4}$ passes through three magnetic transitions upon decreasing temperature $T$. The sinusoidal AF3 phase $(12.6<T<13.5 \mathrm{~K})$ is followed by a noncollinear helical spin phase AF2 $(7.8<T<12.6 \mathrm{~K})$, both phases with an incommensurate (IC) modulation vector $\overrightarrow{q_{2,3}}$ $=(-0.214,1 / 2,0.457)$. The AF1 phase below $7.8 \mathrm{~K}$ is collinear $\left[\vec{q}_{1}=( \pm 1 / 4,1 / 2,1 / 2)\right]$ and shows the typical $\uparrow \uparrow \downarrow \downarrow(E$ type) spin pattern. ${ }^{14}$ Ferroelectricity was only observed in the helical AF2 phase, ${ }^{6,15}$ but Heyer et al. reported a finite value of the FE polarization also in the low temperature AF1 commensurate phase. ${ }^{16}$ However, our recent polarization mea- surements of $\mathrm{MnWO}_{4}$ at ambient and high pressures ${ }^{13}$ are consistent with the paraelectric nature of the AF1 phase. Magnetic frustration due to competing exchange interactions and a strong uniaxial anisotropy lead to the sequence of complex magnetic structures. Tuning the magnetic exchange interactions appears to be imperative to arrive at a deeper understanding of the multiferroic properties of $\mathrm{MnWO}_{4}$ and related compounds. The superexchange interactions depend on the type of magnetic ions, their interatomic distances, and bond angles. The remarkable sensitivity of the FE and magnetic phases of multiferroic materials with respect to hydrostatic pressure was demonstrated for $\mathrm{MnWO}_{4},{ }^{13} \mathrm{Ni}_{3} \mathrm{~V}_{2} \mathrm{O}_{8},{ }^{11}$ and $\mathrm{RMn}_{2} \mathrm{O}_{5},{ }^{12}$ proving the high susceptibility of multiferroics to small perturbations. Alternatively, magnetic exchange interactions can be controlled by replacing one kind of magnetic ions with other ions, either nonmagnetic or with different magnetic properties. In $\mathrm{MnWO}_{4}$, the magnetic $\mathrm{Mn}^{2+}$ ion with spin $S=5 / 2$ can easily be replaced by the $\mathrm{Fe}^{2+}$ ion with a smaller magnetic moment $(S=2)$ and different exchange and anisotropy constants. In an attempt to examine the detailed interplay between magnetic interactions and ferroelectricity, we have therefore investigated the solid solution $\mathrm{Mn}_{1-x} \mathrm{Fe}_{x} \mathrm{WO}_{4}$. We found that $\mathrm{Mn}_{0.9} \mathrm{Fe}_{0.1} \mathrm{WO}_{4}$ is paraelectric $(\mathrm{PE})$ in zero magnetic field at all temperatures; however, ferroelectricity and multiferroic properties are induced by magnetic fields above $4 \mathrm{~T}$.

\section{METHODOLOGY}

Polycrystalline $\mathrm{Mn}_{0.9} \mathrm{Fe}_{0.1} \mathrm{WO}_{4}$ was synthesized by solid state reaction at $930{ }^{\circ} \mathrm{C}$ of the precursor compounds $\mathrm{Mn}_{2} \mathrm{O}_{3}$, $\mathrm{WO}_{3}$, and $\mathrm{Fe}_{2} \mathrm{O}_{3}$, mixed in the appropriate ratios. Single crystals have been grown from the polycrystalline feed rod in a floating zone furnace. X-ray analysis showed the monoclinic structure with lattice constants $a=4.799(2) \AA, b$ $=5.736(2) \AA$, and $c=4.980(2) \AA$, in good agreement with previous reports on natural and synthetic Wolframites. ${ }^{17,18}$ The crystals have been characterized and oriented by single crystal Laue diffractometry. The homogeneity of the crystals and their Fe content were verified by wavelength dispersive spectroscopy analysis. Magnetic measurements were con- 
ducted in a superconducting quantum interference device in magnetic fields up to $5 \mathrm{~T}$. For the dielectric constant and polarization measurements a homemade dielectric probe was adapted to the physical property measurement system (PPMS) for temperature and magnetic field $(H<7 \mathrm{~T})$ control. The crystals were shaped as thin parallel plates, the dielectric constant was calculated from the capacitance measured by the high-precision AH2500A capacitance bridge, and the polarization was determined by integrating the pyroelectric current measured by a K6517A electrometer. During the pyroelectric measurements, a small poling electric field was applied in order to align domains in the ferroelectric state. The PPMS was also used for heat capacity measurements at temperatures above $1.8 \mathrm{~K}$ in different magnetic fields.

\section{EXPERIMENTAL RESULTS}

The low-field magnetization data are consistent with previous reports ${ }^{19}$ and they reveal two successive phase transitions at $T_{N}=15.4 \mathrm{~K}$ and at $T_{L}=12 \mathrm{~K}$, respectively, in excellent agreement with the phase diagram of $\mathrm{Mn}_{1-x} \mathrm{Fe}_{x} \mathrm{WO}_{4}$ for $x=0.1$ previously derived from neutron scattering experiments. ${ }^{18}$ According to the neutron data, magnetic order sets in at $T_{N}$ with an IC modulation vector $\vec{q}_{3}$ $=(-0.235,1 / 2,0.49)$, similar to the AF3 phase of pure $\mathrm{MnWO}_{4}$. At $T_{L}$, the magnetic modulation locks into a commensurate order described by $\vec{q}_{1}=( \pm 1 / 4,1 / 2,1 / 2)$ (AF1 phase of $\mathrm{MnWO}_{4}$ ). The spins are oriented along the easy axis in the $a c$ plane at an angle of $35^{\circ}$ with the $a$ axis.

\section{A. Dielectric constant and polarization}

In order to search for the signature of ferroelectricity, we have performed measurements of the dielectric constant $\varepsilon(T)$ and the pyroelectric current along all three crystallographic orientations. In zero magnetic field, we did not find any indication of a FE transition, in contrast to the ferroelectricity observed in $\mathrm{MnWO}_{4}{ }^{6}$ Minute changes of slope of $\varepsilon(T, H$ $=0)$ near $T_{N}$ and $T_{L}$ are barely visible in the inset of Fig. 1. The replacement of $10 \% \mathrm{Mn}$ by Fe apparently leads to the complete loss of ferroelectricity and, presumably, the loss of the helical magnetic structure in the compound. This conclusion is supported by the results of neutron scattering experiments that did not show an intermediate phase between $T_{N}$ and $T_{L}$ in zero magnetic field.

In external magnetic fields $H_{e}$, oriented along the magnetic easy axis, however, new anomalies in the dielectric properties, measured along the monoclinic $b$ axis, could be observed. In the following we discuss dielectric and polarization data measured along the $b$ axis in external fields $H_{e}$. With increasing magnetic field a distinct peak of $\varepsilon(T)$ develops near the lock-in transition temperature $T_{L}$. The peak appears for fields exceeding about $4 \mathrm{~T}$ and increases with $H_{e}$ (inset of Fig. 1). The pyroelectric current also exhibits a sharp peak, indicating the onset of FE order at $T_{L}$. The results for the polarization $P(T)$ at different magnetic fields are shown in Fig. 1. At $4.5 \mathrm{~T}$, close to $T_{L}, P(T)$ is small but it increases significantly at about $6 \mathrm{~K}$, indicating a major

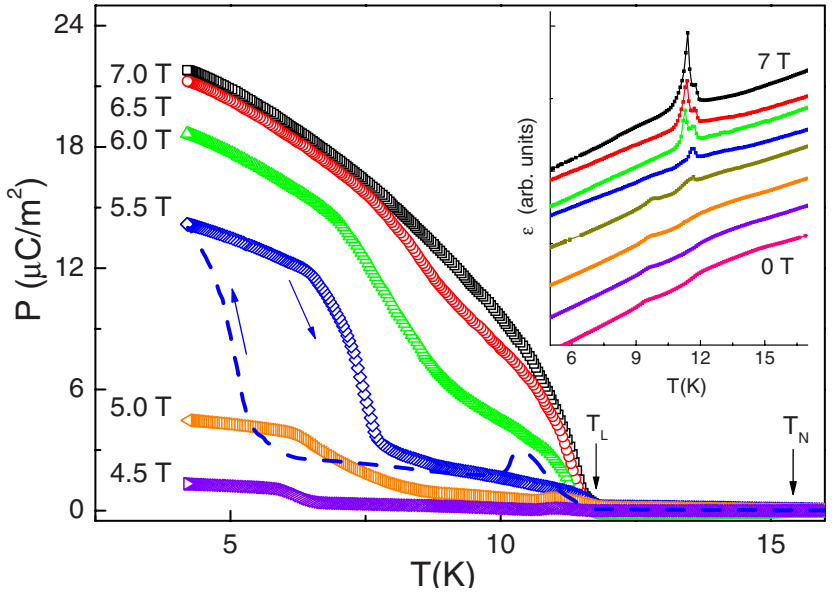

FIG. 1. (Color online) Polarization $P(T)$ of $\mathrm{Mn}_{0.9} \mathrm{Fe}_{0.1} \mathrm{WO}_{4}$ in different fields above $4 \mathrm{~T}$ (cooling data included at $5.5 \mathrm{~T}$ as dashed line, while all other data are shown for increasing $T$ ). Inset: $\varepsilon(T)$ at fields from 0 to $7 \mathrm{~T}$ (curves are vertically offset).

change within the ferroelectric phase from a low-polarization (LP) to a high-polarization (HP) state. Similar transitions within the ferroelectric phase have been observed in other multiferroic compounds and are sometimes attributed to spin reorientations associated with a change of the FE polarization. ${ }^{7}$ The $P(T)$ data also reveal a large temperature hysteresis, as shown for $H_{e}=5.5 \mathrm{~T}$ in Fig. 1. With increasing magnetic field, $P(T)$ increases quickly, and at $7 \mathrm{~T}$, the $\mathrm{FE}$ polarization increases continuously from $T_{L}$ toward lower $T$. From the $\varepsilon(T)$ and $P(T)$ data shown in Fig. 1, we conclude that $\mathrm{Mn}_{0.9} \mathrm{Fe}_{0.1} \mathrm{WO}_{4}$ is paraelectric at zero magnetic field but ferroelectricity is induced by fields above $4 \mathrm{~T}$.

In order to further study the field-induced FE phase, we have conducted field-dependent isothermal polarization measurements. The results (Fig. 2) unambiguously prove the ferroelectricity arising above a critical magnetic field. The transition into the FE phase exhibits a field hysteresis of more than $0.5 \mathrm{~T}$ (inset of Fig. 2) confirming the first-order nature

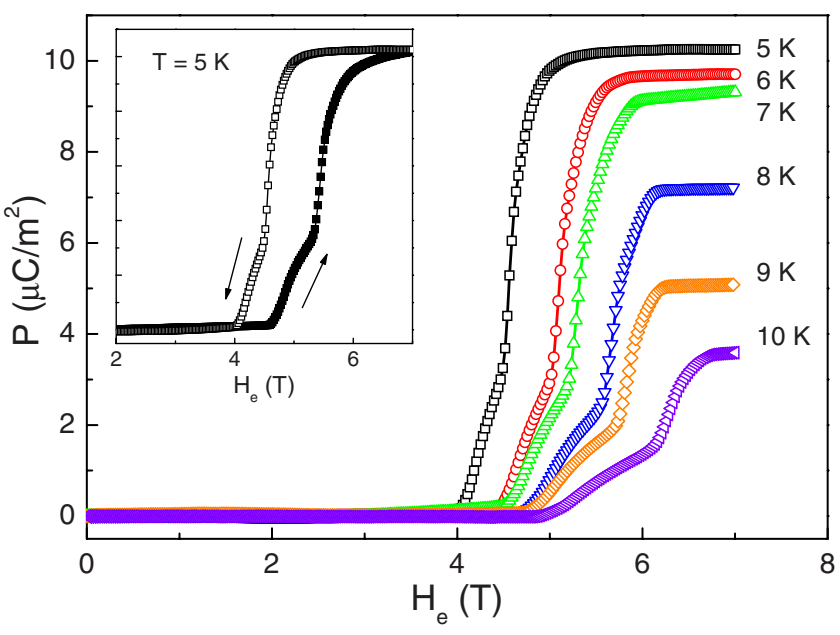

FIG. 2. (Color online) Isothermal field dependence of the FE polarization (data shown for decreasing field only). Inset: $5 \mathrm{~K}$ data with increasing and decreasing field. 


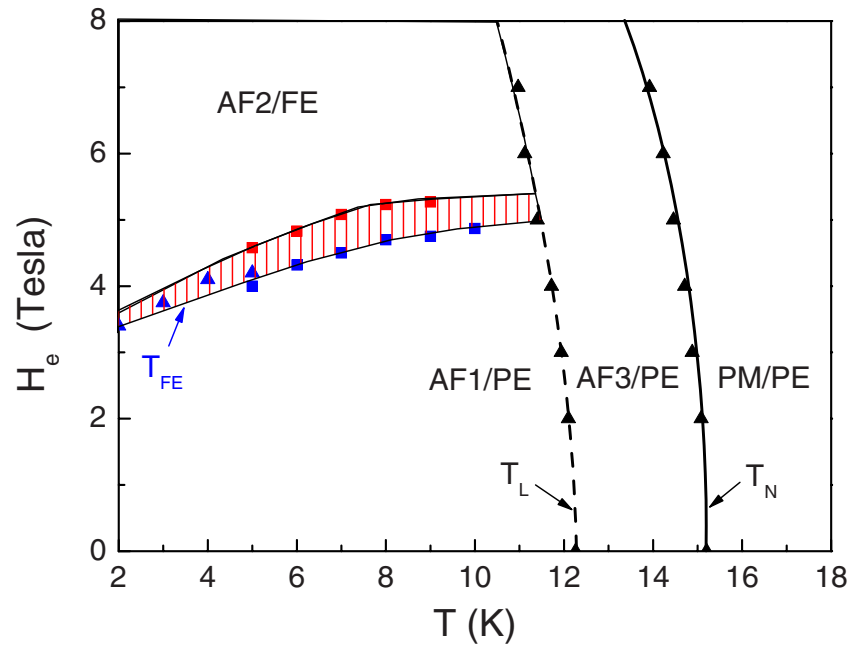

FIG. 3. (Color online) Magnetic phase diagram of $\mathrm{Mn}_{0.9} \mathrm{Fe}_{0.1} \mathrm{WO}_{4}$ with $\mathrm{H}$ along the easy axis. The $\mathrm{FE}$ phase is labeled AF2/FE. Data are from polarization (squares) and magnetization (triangles) measurements. $T_{F E}$ was determined from $P(H)$ and $M(H)$. The shaded area indicates the field hysteresis of the FE transition.

of this phase transition. $P(H)$ shows a characteristic kink which is consistent with the sudden increase of $P(T)$ from the LP to the HP state, as discussed above. The origin of this distinct feature is not clear and has yet to be investigated. From these data, a field-temperature phase diagram for $\mathrm{Mn}_{0.9} \mathrm{Fe}_{0.1} \mathrm{WO}_{4}$ is constructed and shown in Fig. 3 (the hysteresis across the ferroelectric transition is shown as the dashed area). The field dependence of the Néel temperature $\left(T_{N}\right)$ and the lock-in temperature $\left(T_{L}\right)$ are derived from magnetic and heat capacity measurements (discussed below). Both temperatures decrease slightly with $H_{e}$ as expected for an antiferromagnetic (AFM) state. At low fields, $T_{N}$ and $T_{L}$ define the transitions $\mathrm{PM} / \mathrm{PE} \rightarrow \mathrm{AF} 3 / \mathrm{PE} \rightarrow \mathrm{AF} 1 / \mathrm{PE}$. The field-induced FE phase is labeled as AF2/FE.

\section{B. Magnetic properties}

The ferroelectricity in most multiferroic compounds is strongly coupled to the magnetic order. Therefore, any change of the dielectric state should also be detected as anomalies of the magnetic properties. The magnetization measurements of $\mathrm{Mn}_{0.9} \mathrm{Fe}_{0.1} \mathrm{WO}_{4}$ shown in Fig. 4 indeed reflect the major phase transitions observed in dielectric and polarization measurements. At the Nèel temperature $M(T)$ exhibits the characteristic maximum with the onset of IC AFM order. $M(T)$ shows a subtle change with a temperature hysteresis of about $2 \mathrm{~K}$ at $T_{L}$. This hysteresis indicates the first-order nature of the lock-in transition. In high magnetic fields and at low temperature, $M(T)$ reveals another distinct anomaly with a wide hysteresis similar to the polarization data shown in Fig. 1. The change of the magnetic order at the high-field FE transition is also detected in the $M(H)$ measurements (inset of Fig. 4). The sharp increase of $M(H)$ above $4 \mathrm{~T}$ and the observed hysteresis suggest that the magnetic order undergoes a major modification. Note that the

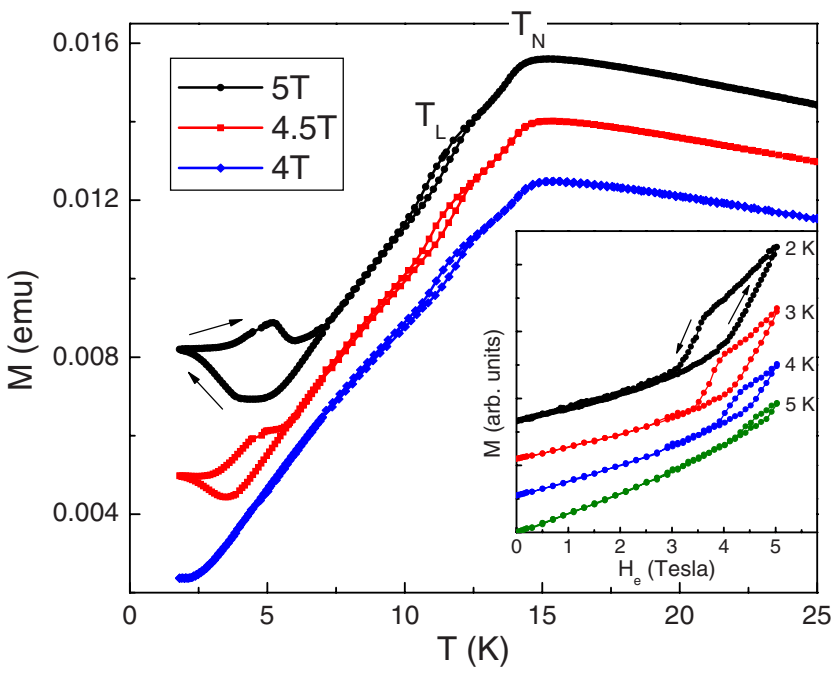

FIG. 4. (Color online) High-field magnetization $M$ of $\mathrm{Mn}_{0.9} \mathrm{Fe}_{0.1} \mathrm{WO}_{4}$. The loop at low $T$ indicates the transition into the AF2/HP phase. Inset: $M\left(H_{e}\right)$ at different temperatures (data for $T$ $<5 \mathrm{~K}$ are vertically shifted).

maximum field of the magnetometer $(5 \mathrm{~T})$ is not enough to complete the transition into the high-field phase; however, the critical fields and temperatures derived from the decreasing field cycle (triangles in Fig. 3) agree well with the similar data from the $P(H)$ measurements (squares in Fig. 3). We conclude that the magnetic field along the easy axis induces a new magnetic order that breaks the spatial inversion symmetry and is compatible with the FE order. Any other direction of the magnetic field was found to be less or not at all efficient in stabilizing the FE phase. We have also extended the pyroelectric measurements along the $a$ and $c$ axes at zero and high magnetic fields but we did not find any signature of FE order along these orientations.

\section{Heat capacity}

The thermodynamic signature of transitions between different phases or orders is usually detected in distinct anomalies of the heat capacity, $C_{p}(T)$. Multiferroic materials with a sequence of subsequent transitions may show more or less pronounced sudden changes of $C_{p}$, some can be subtle and difficult to detect. ${ }^{7}$ Figure 5 shows the heat capacity data, $C_{p} / T$, for $\mathrm{Mn}_{0.9} \mathrm{Fe}_{0.1} \mathrm{WO}_{4}$ in different external magnetic fields $H_{e}$. For $H_{e}=0$, a sharp increase of $C_{p}(T)$ at $T_{N}$ indicates the onset of the IC sinusoidal order. At the lock-in transition, $C_{P}$ exhibits a sharp peak which is difficult to resolve in detail because of the strong first-order nature of this transition. No significant field dependence of $C_{p} / T$ is noted up to $3 \mathrm{~T}$. The only change in this low-field range is a small shift of the steplike increase at $T_{N}$ and the peak at $T_{L}$ toward lower $T$. At higher magnetic fields, however, $C_{p} / T$ shows a well resolved enhancement below the critical temperature of the ferroelectric order (indicated by dashed arrows in Fig. 5). This anomaly quickly shifts to higher $T$ with increasing $H_{e}$ and it merges with the lock-in temperature $T_{L}$ at the highest field (7 T in Fig. 5). The enhanced heat capacity is a clear thermodynamic signature of the ferroelectric order in the 


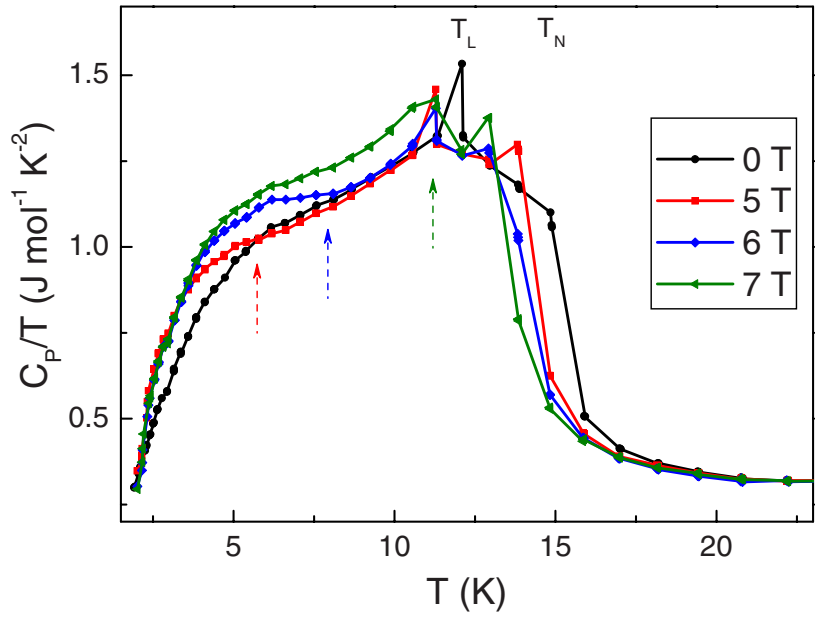

FIG. 5. (Color online) Heat capacity $C_{p} / T$ of $M$ of $\mathrm{Mn}_{0.9} \mathrm{Fe}_{0.1} \mathrm{WO}_{4}$ at zero and high magnetic fields (field along the easy axis). The dashed vertical arrows point to the anomaly associated with the ferroelectric transition at 5, 6, and $7 \mathrm{~T}$, respectively (left to right).

$\mathrm{AF} 2 / \mathrm{FE}$ phase and it proves the bulk nature of the FE highfield phase.

\section{MODEL CALCULATIONS}

Ferroelectricity induced by a magnetic field is a rare phenomenon and it shows the sensitivity of the frustrated magnetic system toward small perturbations. A simple model describing the different magnetic structures (commensurate $q$ $=1 / 4$ at $T=0$, IC helical and sinusoidal orders at $T>0$ ) has to include the competing exchange interactions, the magnetic anisotropy, and the external field $(\vec{H})$. It should be noted that the spin order in the low-temperature commensurate phase of $\mathrm{MnWO}_{4}$ can be represented by a stack of spin chains in the ac plane with equal spin values within each chain but a fourfold modulation $(q=1 / 4)$ of moments perpendicular to the chains. The order of spin chains is then described by the characteristic $\uparrow \uparrow \downarrow \downarrow$ ( $E$ type) modulation. ${ }^{14}$ Within a simplified description, we can describe the major features of the magnetic order by a mean-field model of spin chains stacked along one dimension with effective exchange coupling and anisotropy parameters.

The Heisenberg model with competing nearest $\left(J_{1}\right)$ and next-nearest-neighbor interactions $\left(J_{2}\right)$ and spin anisotropy $(K)$ interpolates between the helical ground state (isotropic model $)^{20}$ and the $E$-type $(\uparrow \uparrow \downarrow \downarrow)$ modulation for strong uniaxial anisotropy (Ising limit). ${ }^{21}$ A similar model has been employed to describe qualitatively the magnetic phase sequence in $\mathrm{Ni}_{3} \mathrm{~V}_{2} \mathrm{O}_{8} \cdot{ }^{22}$ For simplicity, we consider the $\mathrm{S}=1$ model with the easy axis of magnetization along the $z$ direction,

$$
H=J_{1} \sum \vec{S}_{n} \vec{S}_{n+1}+J_{2} \sum \vec{S}_{n} \vec{S}_{n+2}-K \sum\left(S_{n}^{z}\right)^{2}-\vec{H} \sum \vec{S}_{n},
$$

which includes the most basic parameters and interactions to describe the various spin orders in $\mathrm{Mn}_{1-x} \mathrm{Fe}_{x} \mathrm{WO}_{4}$ and other

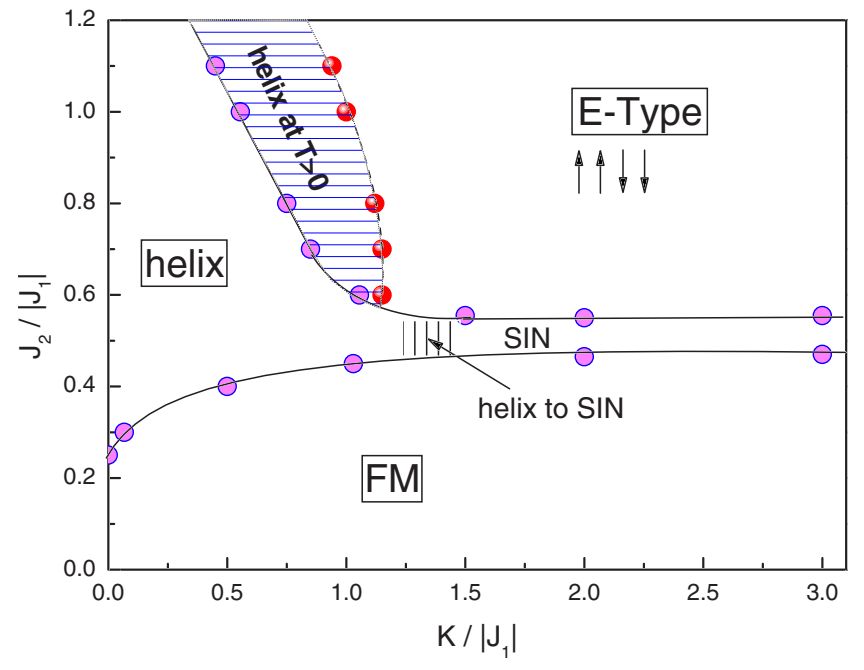

FIG. 6. (Color online) Ground state phase diagram of model (1) in mean-field approximation.

multiferroics. Neutron scattering experiments on $\mathrm{MnWO}_{4}$ suggest a competition between ferromagnetic $(\mathrm{FM})\left(J_{1}<0\right)$ and AFM $\left(J_{2}>0\right)$ exchange interactions and a strong uniaxial anisotropy $(K>0)$, favoring the spin orientation along the easy axis in the $a c$ plane. ${ }^{14}$ Model (1) is solved in the mean-field approximation. In this approximation, the two-spin interaction is replaced by a single-spin term in an effective field of the neighboring spins that is proportional to the corresponding exchange coupling constants and the thermal average of the spins at nearest or next-nearest neighbor sites. The thermodynamic average of all spins is then calculated self-consistently from a set of $N$ coupled equations, with $N$ being the total number of spins along the chain. For simple commensurate spin orders, the set of $N$ equations can be reduced to a smaller number of equations assuming translational symmetry of the average spin values according to the specific order. For example, for a two-sublattice antiferromagnetic order the self-consistency equations are reduced to two coupled equations, one for each sublattice. However, in the case of arbitrary (commensurate or incommensurate) magnetic orders, no assumption about the periodicity of the magnetic order can be made and a large set of $N$ coupled equations has to be considered. We solve the mean-field equations for finite lattices of up to 100 spins assuming periodic boundary conditions. The solution of the mean-field equations for a given set of parameters $\left(K, J_{1}, J_{2}, T\right.$, or magnetic field) without any initial constraints on the magnetic order reproduces the different magnetic structures observed in $\mathrm{Mn}_{1-x} \mathrm{Fe}_{x} \mathrm{WO}_{4}$ and $\mathrm{MnWO}_{4}, E$ type, and sinusoidal (IC) collinear, as well as IC helical spin modulations. Wherever more than one type of order was obtained self-consistently from the equations, the solution with the lower free energy was determined as the stable magnetic configuration.

The ground state phase diagram of Eq. (1) for zero field is shown in Fig. 6. At $K=0$, the ground state is FM for $J_{2} /\left|J_{1}\right|<1 / 4$ and it assumes the noncollinear (NC) incommensurate spin structure for larger $J_{2}$. For large $K$, the Ising limit is reproduced and the transition from FM to the collinear $E$-type magnetic order takes place at $J_{2} /\left|J_{1}\right|=1 / 2$ as in 

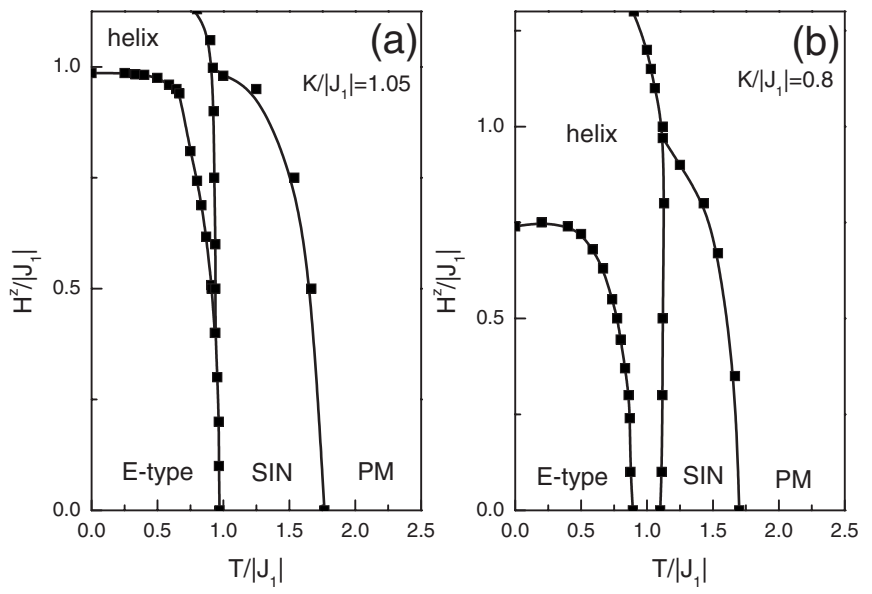

FIG. 7. Field-temperature phase diagrams for model parameters qualitatively describing the phase sequence in (a) $\mathrm{Mn}_{0.9} \mathrm{Fe}_{0.1} \mathrm{WO}_{4}$ and (b) $\mathrm{MnWO}_{4}$.

the Axial Next-Nearest Neighbor Ising model. ${ }^{21}$ The $E$-type phase is stable for larger $J_{2}$ and $K$ and the NC helical phase covers the left section of the ground state phase diagram. For $J_{2} /\left|J_{1}\right| \approx 1 / 2$, the helical spin component perpendicular to the easy axis rapidly decreases with increasing $K$ and it disappears at the transition into the collinear sinusoidal structure close to $K /\left|J_{1}\right| \approx 1.25$ (vertically dashed area in Fig. 6). The horizontally dashed area in Fig. 6 marks the region of special interest. Within this area, the NC helical spin order appears at finite temperatures between the $E$-type ground state and the sinusoidal phase. This phase sequence is typical for $\mathrm{MnWO}_{4}$. With increasing $K$, however, the helical phase becomes unstable at all $T$ and, with increasing temperature, the transition proceeds directly from the $E$-type state to the sinusoidal phase, as observed for $\mathrm{Mn}_{0.9} \mathrm{Fe}_{0.1} \mathrm{WO}_{4}$. The lack of ferroelectricity (at zero field) in $\mathrm{Mn}_{0.9} \mathrm{Fe}_{0.1} \mathrm{WO}_{4}$ is therefore explained by the increase of the uniaxial anisotropy $K$. Evidence for the increase of $K$ with Fe substitution was also derived from the results of neutron scattering experiments. ${ }^{18,19}$ The chosen set of parameters, $J_{2} \approx\left|J_{1}\right|$, results in a spin modulation in the IC phase, $Q_{S I N}=0.21$, that is comparable with the experimental results. ${ }^{14,20}$

In order to understand the magnetic field induced ferroelectricity in $\mathrm{Mn}_{0.9} \mathrm{Fe}_{0.1} \mathrm{WO}_{4}$, we have to consider model (1) in fields oriented along the easy axis ( $z$ axis) and search for NC (helical) spin structures. The solution of Eq. (1) in the mean-field approximation for parameters $J_{1}=-1, J_{2}=1, K$ $=1.05$, and $\vec{H}=\left(0,0, H^{z}\right)$ indeed reveals a magnetic field induced transition from the $E$-type order to a NC helical phase at low temperatures. The $H^{z}-T$ phase diagram for this case is shown in Fig. 7(a). At zero magnetic field the phase sequence with increasing $T$ is $E$ type $\Rightarrow \mathrm{SIN} \Rightarrow \mathrm{PM}$ but above a critical field the helical spin structure is stabilized as an intermediate phase. The helical and the sinusoidal phases are incommensurate with a modulation comparable to the experimental results. It is remarkable that the simple model [Eq. (1)] describes the main physical effects of the Fe sub- stitution, namely, (i) the loss of the FE (helical) phase with the increase of the anisotropy (Fe substitution) and (ii) the reappearance of this phase above a critical magnetic field oriented along the easy axis of magnetization. For lower anisotropy $(K=0.8)$, the known magnetic phase diagram ${ }^{15}$ for $\mathrm{MnWO}_{4}$ is qualitatively reproduced, as shown in Fig. 7(b). Note that the sinusoidal spin order is also suppressed by $\mathrm{H}^{z}$ in agreement with the experimental results for $\mathrm{MnWO}_{4}$. The phase transitions from the $E$ type to sinusoidal and helical phases (Fig. 7) are first-order transitions, whereas a secondorder transition takes place from the sinusoidal to the paramagnetic phase, in accordance with our experimental results discussed above.

\section{SUMMARY AND CONCLUSIONS}

We have shown that the ferroelectric and helical magnetic orders in $\mathrm{MnWO}_{4}$ are easily suppressed by replacing only $10 \%$ of the magnetic $\mathrm{Mn}^{2+}$ ion by another magnetic ion, $\mathrm{Fe}^{2+}$. The ferroelectric state is restored in external magnetic fields applied along the easy axis of the spins. We have resolved the field-temperature phase diagram for $\mathrm{Mn}_{0.9} \mathrm{Fe}_{0.1} \mathrm{WO}_{4}$ showing three distinct magnetic and/or ferroelectric phases. The experimental observations are explained by a mean-field solution of the Heisenberg model with competing interactions, uniaxial anisotropy, and external magnetic field. The destruction of the ferroelectric and/or helical phase is due to an increase of the uniaxial anisotropy with the iron substitution. The calculated phase diagrams describe the experimental results for $\mathrm{Mn}_{0.9} \mathrm{Fe}_{0.1} \mathrm{WO}_{4}$ and $\mathrm{MnWO}_{4}$ qualitatively well. While the model is too simple to allow for any quantitative comparison with the experiments, it includes the major physical interactions to describe qualitatively the different magnetic phases in $\mathrm{MnWO}_{4}$ and $\mathrm{Mn}_{0.9} \mathrm{Fe}_{0.1} \mathrm{WO}_{4}$. Improvements of the model description should include the three-dimensional lattice structure and spin order, the differences in the exchange coupling constants between $\mathrm{Mn}$ and $\mathrm{Fe}$ ions, and the effects of disorder. The results of our simple model calculation should also be relevant to other multiferroic materials such as $\mathrm{CuFeO}_{2}$ where magnetic field induced ferroelectricity has been observed very recently. ${ }^{23,24}$ Our experimental results and calculations for $\mathrm{Mn}_{0.9} \mathrm{Fe}_{0.1} \mathrm{WO}_{4}$ suggest that with increasing magnetic field the collinear $E$-type spin modulation turns into a helical IC magnetic order breaking the spatial inversion symmetry which allows for the observed ferroelectricity. This transition could be verified by high-field neutron scattering experiments. It is expected that these experiments also reveal additional details of the magnetic orders within the FE phase as observed by us in $\mathrm{Mn}_{0.9} \mathrm{Fe}_{0.1} \mathrm{WO}_{4}$. Large single crystals are currently being grown for further investigations.

\section{ACKNOWLEDGMENTS}

This work was supported in part by the T. L. L. Temple Foundation, the J. J. and R. Moores Endowment, and the State of Texas through TCSUH, and at LBNL through the U.S. DOE under Contract No. DE-AC03-76SF00098. 
${ }^{1}$ N. A. Spaldin and M. Fiebig, Science 309, 391 (2005).

${ }^{2}$ Y. Tokura, J. Magn. Magn. Mater. 310, 1145 (2007).

${ }^{3}$ M. Mostovoy, Phys. Rev. Lett. 96, 067601 (2006).

${ }^{4}$ M. Kenzelmann, A. B. Harris, S. Jonas, C. Broholm, J. Schefer, S. B. Kim, C. L. Zhang, S.-W. Cheong, O. P. Vajk, and J. W. Lynn, Phys. Rev. Lett. 95, 087206 (2005).

${ }^{5}$ G. Lawes, A. B. Harris, T. Kimura, N. Rogado, R. J. Cava, A. Aharony, O. Entin-Wohlman, T. Yildrim, M. Kenzelmann, C. Broholm, and A. P. Ramirez, Phys. Rev. Lett. 95, 087205 (2005).

${ }^{6}$ K. Taniguchi, N. Abe, T. Takenobu, Y. Iwasa, and T. Arima, Phys. Rev. Lett. 97, 097203 (2006).

${ }^{7}$ C. R. dela Cruz, F. Yen, B. Lorenz, M. M. Gospodinov, C. W. Chu, W. Ratcliff, J. W. Lynn, S. Park, and S.-W. Cheong, Phys. Rev. B 73, 100406(R) (2006).

${ }^{8}$ D. Higashiyama, S. Miyasaka, N. Kida, T. Arima, and Y. Tokura, Phys. Rev. B 70, 174405 (2004).

${ }^{9}$ N. Hur, S. Park, P. A. Sharma, J. S. Ahn, S. Guha, and S.-W. Cheong, Nature (London) 429, 392 (2004).

${ }^{10}$ C. R. dela Cruz, B. Lorenz, Y. Y. Sun, C. W. Chu, S. Park, and S.-W. Cheong, Phys. Rev. B 74, 180402(R) (2006).

${ }^{11}$ R. P. Chaudhury, F. Yen, C. R. dela Cruz, B. Lorenz, Y. Q. Wang, Y. Y. Sun, and C. W. Chu, Phys. Rev. B 75, 012407 (2007).

${ }^{12}$ C. R. dela Cruz, B. Lorenz, Y. Y. Sun, Y. Wang, S. Park, S.-W. Cheong, M. M. Gospodinov, and C. W. Chu, Phys. Rev. B 76, 174106 (2007).

${ }^{13}$ R. P. Chaudhury, F. Yen, C. R. dela Cruz, B. Lorenz, Y. Q. Wang,
Y. Y. Sun, and C. W. Chu, arXiv:0705.4675, Physica B 403, 1428 (2008).

${ }^{14}$ G. Lautenschläger, H. Weitzel, T. Vogt, R. Hock, A. Bohm, M. Bonnet, and H. Fuess, Phys. Rev. B 48, 6087 (1993).

${ }^{15}$ A. H. Arkenbout, T. T. M. Palstra, T. Siegrist, and T. Kimura, Phys. Rev. B 74, 184431 (2006).

${ }^{16}$ O. Heyer, N. Hollmann, I. Klassen, S. Jodlauk, L. Bohaty, P. Becker, J. A. Mydosh, T. Lorenz, and D. Khomskii, J. Phys.: Condens. Matter 18, L471 (2006).

${ }^{17}$ R. Guillen and J. R. Regnard, Phys. Chem. Miner. 12, 246 (1985).

${ }^{18}$ E. Garcia-Matres, N. Stüßer, M. Hofmann, and M. Reehuis, Eur. Phys. J. B 32, 35 (2003).

${ }^{19}$ N. Stüßer, Y. Ding, M. Hofmann, M. Reehuis, B. Ouladdiaf, G. Ehlers, D. Günther, M. Meißner, and M. Steiner, J. Phys.: Condens. Matter 13, 2753 (2001).

${ }^{20}$ T. Nagamiya, Solid State Phys. 20, 305 (1967).

${ }^{21}$ P. Bak, Rep. Prog. Phys. 45, 587 (1982).

${ }^{22}$ M. Kenzelmann, A. B. Harris, A. Aharony, O. Entin-Wohlman, T. Yildirim, Q. Huang, S. Park, G. Lawes, C. Broholm, N. Rogado, R. J. Cava, K. H. Kim, G. Jorge, and A. P. Ramirez, Phys. Rev. B 74, 014429 (2006).

${ }^{23}$ T. Kimura, J. C. Lashley, and A. P. Ramirez, Phys. Rev. B 73, 220401(R) (2006).

${ }^{24}$ S. Kanetsuki, S. Mitsuda, T. Nakajima, D. Anazawa, H. A. Katori, and K. Prokes, J. Phys.: Condens. Matter 19, 145244 (2007). 